\title{
Adaptive Optics for space debris tracking
}

\author{
Francis Bennet $^{a}$, Céline D’Orgeville ${ }^{a}$, Yue Gao ${ }^{b}$, William Gardhouse $^{a}$, Nicolas Paulin ${ }^{a}$, Ian \\ Price $^{a}$, François Rigaut ${ }^{a}$ Ian Ritchie ${ }^{b}$, Craig Smith $^{b}$, Kristina Uhlendorf ${ }^{a}$, Yanjie Wang ${ }^{b}$ \\ ${ }^{a}$ Research School of Astronomy and Astrophysics, Australian National University, Advanced \\ Instrumentation \& Technology Centre, Canberra, Australia \\ ${ }^{b}$ EOS Space Systems, Mount Stromlo Observatory, Canberra, Australia
}

\begin{abstract}
Space debris in Low Earth Orbit (LEO) is becoming an increasing threat to satellite and spacecraft. A reliable and cost effective method for detecting possible collisions between orbiting objects is required to prevent an exponential growth in the number of debris. Current RADAR survey technologies used to monitor the orbits of thousands of space debris objects are relied upon to manoeuvre operational satellites to prevent possible collisions. A complimentary technique, ground-based laser LIDAR (Light Detection and Ranging) have been used to track much smaller objects with higher accuracy than RADAR, giving greater prediction of possible collisions and avoiding unnecessary manoeuvring. Adaptive optics will play a key role in any ground based LIDAR tracking system as a cost effective way of utilising smaller ground stations or less powerful lasers. The use of high power and high energy lasers for the orbital modification of debris objects will also require an adaptive optic system to achieve the high photon intensity on the target required for photon momentum transfer and laser ablation.

EOS Space Systems have pioneered the development of automated laser space debris tracking for objects in low Earth orbit. The Australian National University have been developing an adaptive optics system to improve this space debris tracking capability at the EOS Space Systems Mount Stromlo facility in Canberra, Australia. The system is integrated with the telescope and commissioned as an NGS AO system before moving on to LGS $\mathrm{AO}$ and tracking operations. A pulsed laser propagated through the telescope is used to range the target using time of flight data. Adaptive optics is used to increase the maximum range and number or targets available to the LIDAR system, by correcting the uplink laser beam.

Such a system presents some unique challenges for adaptive optics: high power lasers reflecting off deformable mirrors, high slew rate tracking, and variable off-axis tracking correction. A low latency real time computer system is utilised to control the systems, with a Shack-Hartmann wavefront sensor and deformable mirror running at 1500 frames per second. A laser guide star is used to probe the atmosphere and the tracked debris object is used as a natural guide star for tip-tilt correction.
\end{abstract}

Keywords: Adaptive Optics, Space Environment Management, Laser Ranging

Further author information: (Send correspondence to F. Bennet.)

F. Bennet.: E-mail: francis.bennet@anu.edu.au

Adaptive Optics Systems IV, edited by Enrico Marchetti, Laird M. Close,

Jean-Pierre Véran, Proc. of SPIE Vol. 9148, 91481F · @ 2014 SPIE

CCC code: $0277-786 \mathrm{X} / 14 / \$ 18 \cdot$ doi: $10.1117 / 12.2055560$

Proc. of SPIE Vol. $914891481 \mathrm{~F}-1$ 


\section{INTRODUCTION}

Advances in technology throughout human history have worked to improve our lives and enrich our society. Our advance beyond Earth into space has marked a technological revolution and plays a curtail role in our modern lives. Since the first satellite ascended into orbit in 1957 we have been launching more and more objects into orbit every year.

The persistent nature of an orbit makes space the ideal location to place valuable assets capable of connecting continents. This persistence also causes a major problem for these satellites as any fragment of past rocket launch or defunct satellite have the capability to severely damage or destroy otherwise perfectly functional and valuable pieces of equipment. ${ }^{1}$ Objects in Low Earth Orbit (LEO) can remain there for years or decades before the orbit decays enough to reenter the atmosphere, providing a long life for functioning satellites to complete their mission. Ideally any debris objects would be returned to Earth shortly after being discarded, however most of the debris will end up in the most populated and efficient orbits. In order to be cost effective a single satellite's useful lifetime must span many years if not decades, during which time the satellite must be kept in an operational state. Orbiting space debris must therefore be tracked accurately to protect satellites, and measures must be taken to minimise the creation of new debris objects.

The Research School of Astronomy and Astrophysics (RSAA) at the Australian National University located at the Mount Stromlo Observatory in Canberra, Australia, have been developing an Adaptive Optic (AO) system to improve the tracking capability of ground based telescopes in partnership with Electro Optic Space Systems (EOS). This Adaptive Optic Demonstrator (AOD) project aims to build and demonstrate a Laser Guide Star (LGS) based AO system with a $1.8 \mathrm{~m}$ diameter telescope based at the Mount Stromlo Observatory.

\subsection{Space Debris}

Space debris can be classified as any man made object in orbit which no longer serves a useful purpose. A piece of space debris can anything from a spent rocket stage, unspent propellant, material such as flecks of paint from the degradation and corrosion of other objects, and anything else originating from man made objects in orbit. The vast majority of the debris is less than $1 \mathrm{~cm}$ in diameter and pose significant risk to any asset in orbit. A small number large debris objects $(>10 \mathrm{~cm})$ are regularly tracked by ground based RADAR to provide some assistance to satellite operators in avoiding possible catastrophic collisions.

Most space debris occur in the highly populated communication satellite polar orbits between altitudes of 750 and $800 \mathrm{~km}$, and nearly all the debris is contained within 2,000 km of Earth. The $800 \mathrm{~km}$ orbit is favoured because it minimises the launch cost and atmospheric drag, meaning satellites can remain in a stable orbit for decades with minimal fuel expenditure. This also means that debris will remain in these orbits for a similarly lengthy period.

Several anti-satellite weapons tests ${ }^{2}$ and the collision between the Iridium 33 and Cosmos $2251^{3}$ satellites between 2007 and 2009 have drastically increased the number of fragmentation debris formed by collisions. The increased density of debris objects will accelerate the instability of LEO, ${ }^{4}$ necessitating the development of practical debris tracking and management strategies such as using a tether and space tug, ${ }^{5}$ and using laser ablation or photon pressure to de-orbit debris ${ }^{3,6-10}$ for continued safe operations in LEO.

\subsection{Current Mitigation Strategies}

While many rockets launched by private and government organisations today minimise the generation of space debris, there are no international standard guidelines or treaties governing the generation of space debris. Most major space capable nations (e.g. Unite States, Russia, China, Japan, European Space Agency) have guidelines to minimise space debris generation. These strategies include leaving spent boost stages in low orbits to speed up the reentry of any debris generated with the launch. Valuable geostationary orbits are protected by moving satellites to a higher 'graveyard' orbit where they will pose minimal risk to any future satellites. In order to stabilise the LEO environment several large objects should be removed from orbit each year, ${ }^{11}$ combined with lowering the impact of new satellite launches. 


\subsection{Space Environment Management}

A space environment management strategy must be developed or help maintain the viability of satellite networks. As the space above Earth becomes more crowded the chance of more catastrophic collisions increases exponentially, and is known as the Kessler syndrome. ${ }^{1}$ Relative velocities between two orbits can easily exceed 10s of kilometres per second, and any resultant collisions produce a large number of new debris objects, resulting in a cascading increase in debris objects. ${ }^{1}$

A recently founded Australian Research Council supported Cooperative Research Centre (CRC) for Space Environment Management brings together research and industry partners from around the world to develop tracking and orbital modification methods using ground based telescopes. The Space Environment Management CRC brings together partners from The Australian National University, Lockheed Martin, EOS Space Systems, NASA Ames Research Center, National Institute of Information and Communications Technology, Optus, and RMIT University. The goals of the Space Environment Management CRC include tracking space debris more often and more accurately, improving predictions of space debris orbits, and predict and monitor potential collisions in space. The partners all bring expertise and resources from leading universities, commercial research centres and space agencies. The information and technology developed will be used to help manage the space environment by reducing collisions and debris population.

\section{CURRENT TRACKING TECHNOLOGIES}

The United States Space Surveillance Network maintains a database of space debris objects larger than 10 $\mathrm{cm}$. Objects in LEO are routinely tracked using RADAR, while optical telescopes are used to track larger objects which are further away. Optical telescopes are more efficient at tracking these more distant objects because the sunlight reflecting off them only suffers a $1 / r^{2}$ drop in intensity for a range to target of $r$. Since radio telescopes must provide their own illumination source, the received power suffers a $1 / r^{4}$ drop in intensity, reducing its efficiency for higher orbits. While these two techniques provide valuable information on space debris and operational satellites, the growing density of assets in highly valuable orbits require a more accurate method of tracking both space debris and operational satellites. Active ground based tracking using laser illumination and ranging can provide highly accurate LIDAR information which can be used in sophisticated orbital prediction algorithms to project an objects position with an extremely high degree of accuracy.

\subsection{RADAR and Optical Tracking}

Radio Detection and Ranging (RADAR) has been used since its development in the early 1900s to detect and range objects at great distance. The technology consists of a radio transmitter and receiver, along with signal processing electronics to amplify and interpret received signals. RADAR is particularly useful for tracking orbiting objects ${ }^{12}$ as the conductive materials they are constructed from are highly reflective in the microwave and radio spectrum. Typical wavelengths range from $2 \mathrm{~cm}$ to $6 \mathrm{~m}$, have the advantage of working in most weather conditions, and most wavelengths have good transmission through the atmosphere. The wavelength does however limit the accuracy and size of objects which can be tracked, and available transmitter power provides a maximum range.

Optical tracking falls into one of two categories, passive tracking in which reflected light is used to track and object, or active tracking, where a light source is projected onto the target, and the reflection used for tracking. Passive optical tracking can track objects further away, provided they are illuminated by the sun and provide more light than the sky background, limiting tracking observations to the night. This limits LEO tracking to a few hours after dusk and before dawn, while objects in higher orbits can be tracked at any time of the night. While the wavelength of light $\left(\sim 10^{-6} \mathrm{~m}\right)$ can in theory provide highly accurate orbital information, refraction by the atmosphere in the form of atmospheric turbulence can distort the image and position of the object quite significantly. Atmospheric absorption also limits the wavelength range available to the visible and near infrared. Optical tracking is also limited in accuracy and object size by the amount of reflected light from the object. Small and low albedo objects may not reflect enough light to provide a good signal to noise ratio for the telescope, which can results in inaccurate measurements.

An active optical debris and satellite tracking system can be made using a combination of optical and RADAR techniques, known as LIDAR. A LIDAR system illuminates a target with a laser beam, and measures the range 
to the target by accurately timing the round trip of a single laser pulse. This eliminates the need for sunlit objects, improving the minimum size and altitude to which object can be tracked. A ranging system also needs to be able to measure the distance from the ground station to the target, as opposed to a tracking station which determines an orbit by tracking the object multiple times and potentially with different ground stations. A laser beam can be collimated or focused for a specific range or altitude to maximise the return signal from a single target, rather than a broad beam produced by a radio transmitter. The accuracy is determined primarily by the wavelength of the probing beam (for example $1064 \mathrm{~nm}$ ). Ranging accuracy can be as low as a few centimetres with a visible or infra-red laser system. This allows for better orbital prediction than can be achieved with much longer wavelengths used by RADAR systems.

LIDAR can be used to track both uncooperative targets such as space debris, and cooperative targets which are satellites fitted with retro-reflectors. The size of the ground based telescope will limit the reflected light collected, limiting both the size and range of trackable targets. Cooperative targets are tracked to refine their orbit, and some satellites in highly stable orbits are used for calibration for tracking systems.

\section{ADAPTIVE OPTICS DEMONSTRATOR FOR TRACKING SPACE DEBRIS}

The Adaptive Optic Demonstrator (AOD) is a partnership between the RSAA and EOS at the Mount Stromlo Observatory. The AOD project is used here as an example of how AO can improve satellite and space debris tracking. The project's goals are to demonstrate the improvement to tracking capability of a ground based active optical tracking system with AO. The delivered higher beam quality of an AO system will allow the system to track debris at greater range, as well as smaller objects. The higher quality beam will vastly increase the population of LEO objects available to track because the population of space debris is dominated by objects less than $10 \mathrm{~cm}$ in diameter, and so any increase in sensitivity for smaller objects will provide the largest increase to tracking productivity. This allows LIDAR systems to compliment existing RADAR tracking programs, increasing both the accuracy and number of objects in debris catalogues. The project utilises AO and instrumentation expertise at RSAA and the tracking facilities of EOS. The system consists of a $1064 \mathrm{~nm} 200 \mathrm{~W}$ nanosecond pulsed laser system and a $1.8 \mathrm{~m}$ telescope, with a laser guide star adaptive optic system.

The laser propagates from an optical bench through the AO system to the telescope, and a send/receive chopper wheel separates the sent and received laser pulses. The AO system contains a Shack-Hartmann wavefront sensor, a tip-tilt sensor and a deformable mirror. The tip-tilt sensor also serves as a telescope acquisition and tracking system. The ranging is performed by timing a sent pulse from the laser. The orbit is then determined by the track performed by the telescope to follow the target.

\subsection{EOS Laser Ranging}

EOS have been developing active satellite and debris tracking, with a system capable of determining an orbit accurately, and predicting a debris objects position to within $200 \mathrm{~m}$ after 24 hours. ${ }^{6}$ This is an order of magnitude better than can be achieved with RADAR or passive optical systems, because the active tracking of orbiting objects also includes a range component accurate to a few meters, allowing for more accurate orbital determination. EOS have been able to track debris objects as small as $10 \mathrm{~cm}$ to a range of $2000 \mathrm{~km}$.

EOS have two main facilities at the Mount Stromlo Observatory, an automated cooperative target ranging system, and a debris ranging system for uncooperative objects lacking retro reflectors. The automated system uses a $532 \mathrm{~nm}$ pulsed laser emitted and collected by a $1 \mathrm{~m}$ telescope to track and range satellites fitted with retro-reflectors, giving highly accurate orbital determination. The debris ranging system uses a $200 \mathrm{~W} 1064 \mathrm{~nm}$ pulsed laser emitted and collected by a $1.8 \mathrm{~m}$ telescope, and tracks objects which are not fitted with specific reflecting items, including the majority of space debris objects. This system can observe much smaller objects than the satellite tracking system, because of the higher laser power and larger telescope. The system is also currently being upgraded to accept a $1 \mathrm{~kW} 1064 \mathrm{~nm}$ laser system which will be used to 'spotlight' search for debris objects by scanning a broad beam across the sky and looking for return signals. 


\subsection{AO Corrected Laser Ranging}

Actively tracking debris objects using ground based telescopes is currently the most accurate way of tracking space debris. The major limitation on this system today is the ability to illuminate the target with high flux, thereby providing a good signal to noise ratio for ranging of objects below $15 \mathrm{~cm}$ size or beyond $1500 \mathrm{~km}$ in distance. This has three basic causes: laser power, telescope size, and low Strehl ratio caused by atmospheric turbulence. Lasers currently in development can achieve kilowatt power levels with good beam quality, and even a modest size telescope can achieve a diffraction limit small enough to focus laser energy onto a small $(\sim 1 \mathrm{~m})$ area. An AO system can be used to increase the Strehl ratio of the laser beam on the target, providing the final piece to achieving a higher photon return and tracking capacity.

The non-sidereal tracking required to follow orbiting objects results in high slew speeds, particularly close to Zenith. This increases the turbulence seen by the telescope and AO system, resulting in a requirement to run the system very fast.

An AO complimented ranging system uses the same telescope to project and collect reflected laser light to some share critical optical components such as the deformable mirror, and the high laser power requires some precautions not to damage the delicate detectors of the wavefront sensing system. For the AOD project, laser light is expanded and propagated to the deformable mirror via a dichroic beam splitter. Off-axis reflective beam expanders are used to avoid focusing the laser beam because the $200 \mathrm{~W}$ laser used will ionise the atmosphere at a focus, scattering the laser light and reducing the output power significantly. The deformable mirror is made by Xinetics and has 177 actuators in a square $14 \times 14$ grid over a $98 \mathrm{~mm}$ pupil. The beam is further expanded and coupled to the Coudè path to the telescope. Returning light follows the same path and a send/receive box separates sent and received laser pulses. This mechanism consists of a rotating highly polished Aluminium disc with an elliptical hole, through which pulses are sent. Received pulses are reflected from the disc as it spins and passed to a photo-multiplier for detection.

While the proposition of $\mathrm{AO}$ correcting an upward propagating laser beam to track an object in orbit at first seems simple, the details make it quite a complex operation. The refraction of light varies across the satellite track, as does its range and apparent speed. The distances of several hundred kilometres requires the speed of light to be taken into consideration, which means that each laser beam and reflected light source will need to be off-axis with respect to the telescope axis, oriented along the target track to keep the laser beam illuminating the target, the LGS probing the correct portion of atmosphere, and the tip-tilt camera imaging the target. Fig. 1 shows a schematic of the target orbiting the Earth, with the orbit passing overhead the tracking station. The LGS is pointed ahead to intersect the region of atmosphere which will be traversed by the probe laser pulse. By the time the laser pulse reaches the correct altitude the target will have moved to intersect it, and the reflected sun and laser light travels back down to the ground station and appear off-axis.

The probe laser is on the optical axis of the telescope, while the reflected laser light and sunlight used for tiptilt are off-axis. Reflected probe laser light is reflected by the dichroic and directed to the send/return splitting box for ranging detection. Light from the LGS and reflected sunlight from the target are passed through the laser reflecting dichroic to the wavefront sensing subsystem.

\subsection{Wavefront Sensing}

The wavefront sensing subsystem contains a Shack-Hartmann wavefront sensor and a tip-tilt and acquisition camera. The Shack-Hartmann consists of a lenslet array producing $15 \times 15$ subaperatures over the pupil, and uses an OCAM2 detector running at $1.5 \mathrm{kHz}$. Several OD6 $1064 \mathrm{~nm}$ notch filters and a light tight box are used to eliminate any scattered $1064 \mathrm{~nm}$ laser light, to prevent contamination of the LGS and tip-tilt signals. Focus is removed from the DM commands and sent to the LGS facility to maintain focus on sky. An open loop control is used to focus the Shack-Hartmann using a trombone mechanism, on the expected LGS altitude for the current telescope elevation. Fig. 2 shows an optical schematic of the wavefront sensing sub system. All of the light collected by the telescope is passed through a beam expander and onto the deformable mirror, where the incoming and outgoing wavefronts are corrected. The incoming light in the visible is passed through a dichroic and onto the wavefront sensors. The $589 \mathrm{~nm}$ LGS light is directed onto the SH-WFS through the focus compensating trombone, and the remainder is passed to the tip-tilt detector. The outgoing probe laser joins 


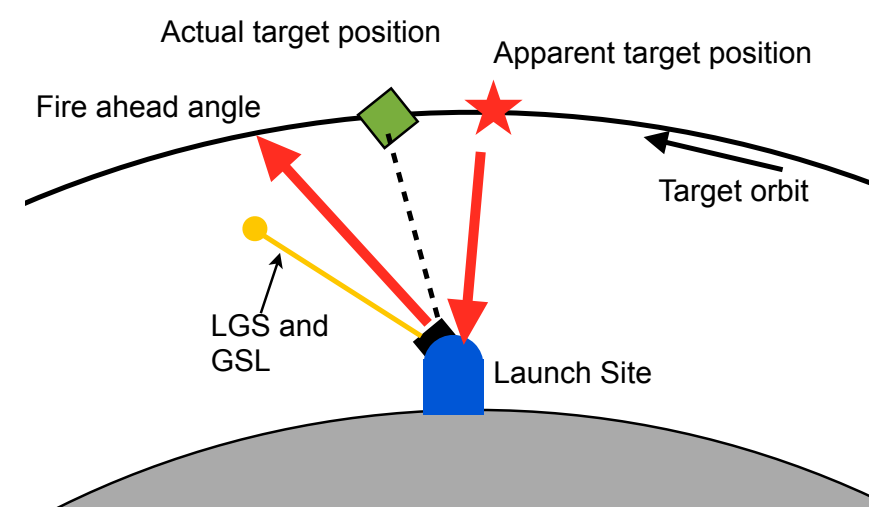

Figure 1. A schematic showing the target in orbit around Earth, passing overhead the ground station for tracking. The GSL is pointed ahead of the laser, such that the LGS samples the atmosphere through which the probe laser is propagated. The probe laser is angled ahead of the actual target position such that a pulse from the laser will intersect with the targets orbit at the correct time. The reflected laser light appears off-axis in the apparent target position, and reflected sunlight is used here for tip-tilt.

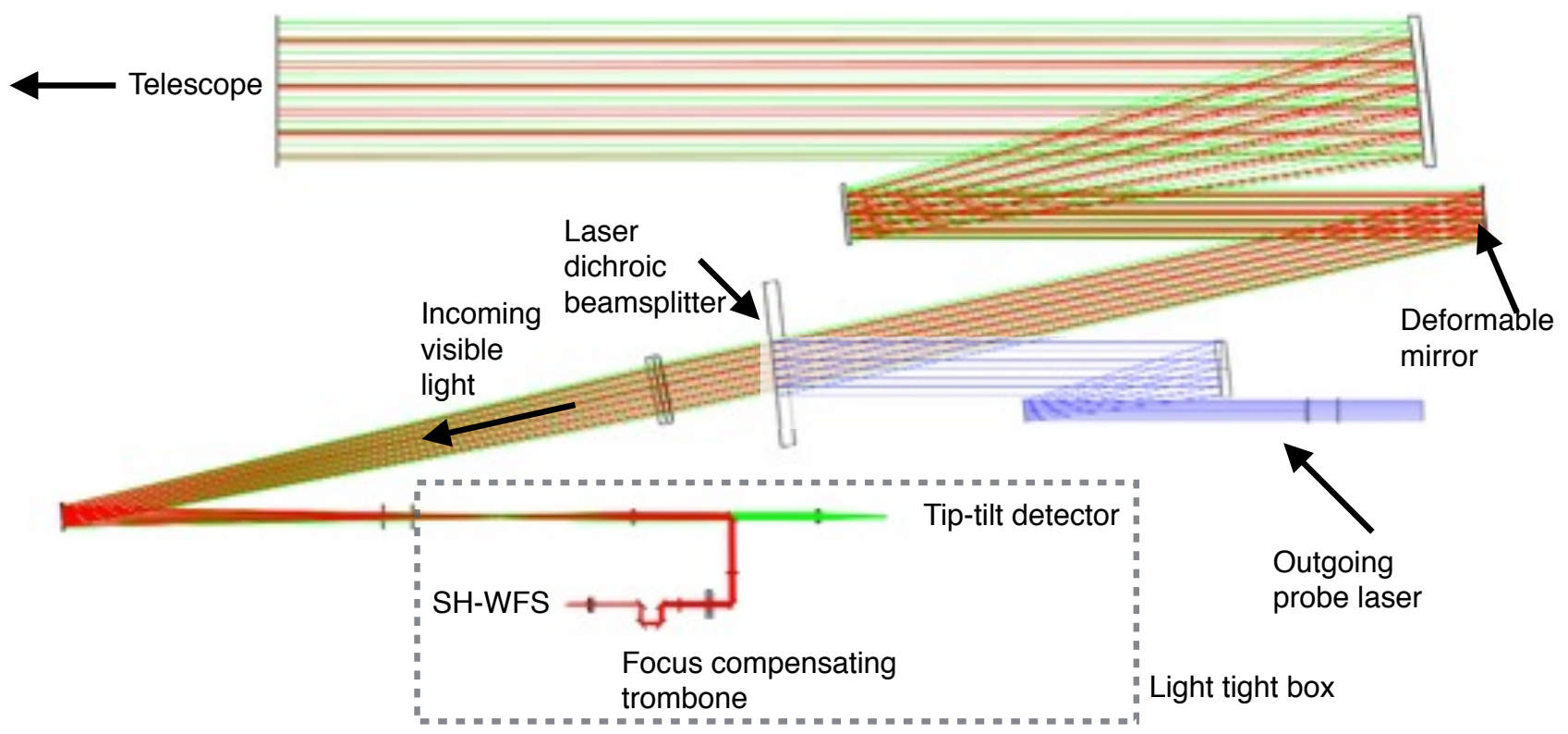

Figure 2. An optical schematic of the wavefront sensing sub system. Light from the telescope passes through a beam expander and onto the deformable mirror, and reflected to the SH-WFS and tip-tilt detector within a light tight box. The outgoing probe laser is combined to the optical path with a dichroic, and the corrected laser pulse travels back through the optical system to the telescope.

the optical path at the laser dichroic and is corrected by the deformable mirror before being directed through the optical system and telescope to the target. Returning laser pulses are also reflected by this dichroic and are passed to the laser system for separation from the outgoing pulses.

The tip-tilt sensor also serves as a telescope tracking and acquisition imager. It consists of an Andor 860 detector imaging the tracked object. The tip-tilt loop operates at $500 \mathrm{~Hz}$ with a leaky integrator driving a tip-tilt stage on the DM. Large scale tip-tilt is offloaded to the telescope as a bias on its track. The guide star is sampled at 0.28 arc seconds per pixel with a field of view of 35 arc seconds. The tip-tilt image is off-axis and will precess around the optical axis. The position of the guide star is known from the telescope track and is used to calculate the tip-tilt. 


\subsection{Laser Guide Star Facility}

The Laser System is a $30 \mathrm{~W}, 589 \mathrm{~nm}$, sum-frequency CW laser locked on the peak of the sodium D2a line with $10 \%$ repumping on the sodium D2b line. The laser bench and associated electronics are housed in a thermally controlled, semi-clean, Laser Service Enclosure (LSE). The LSE is custom-built and retrofitted on the dome third floor to be rotating in azimuth with the EOS telescope. The laser beam is transferred to an off-axis Laser Launch Telescope (LLT) via the Nasmyth port and some Beam Transfer Optics providing active pointing and focus of the Laser Guide Star (LGS) on the sky. The LLT is a $280 \mathrm{~mm}$-aperture refractive beam expander adapted from an earlier, fiber-fed telescope initially built by EOS.

The Laser System for this project is being developed by EOS, while the Beam Transfer Optics (BTO) and Laser Launch Telescope are being developed jointly by EOS and the ANU. Please refer to reference ${ }^{13}$ in these proceedings for details on the LGS Facility design, fabrication status and commissioning plans.

\section{FUTURE DEVELOPMENT}

Active tracking technologies are always limited by the collecting area and coupled illumination power. While large telescopes and power laser systems are expensive, they may prove to be the most cost effective means of tracking orbiting objects because the technology is well developed for astronomical applications, and several ground stations could conceivably track the vast majority of hazardous debris objects. The most pressing concern currently is tracking sufficient orbiting objects accurately enough to predict with a high degree of certainty any possible collisions. This will greatly assist in the prevention of the Kessler syndrome of exponential growth in space debris. Further mitigation measures which may now be too expensive to consider can then be implemented to begin reducing the number of debris in orbit.

One possibility to control the growth of space debris is to capture and return larger objects such as spent rocket stages or inoperable satellites, by capturing them with another space craft or satellite and placing them on a high-drag quickly decaying orbit. While this may be feasible for large space debris, the majority of debris is made up of objects $<10 \mathrm{~cm}$ in size. These objects would prove difficult if not impossible to remove from orbit both because of their size and number.

A ground based approach to management and removal of space debris is to use photon pressure to modify the orbit of a target. A high power ground based laser will most certainly require an AO system to achieve the flux on target necessary to apply enough pressure to shift the orbit a measurable and significant amount. As an initial stage to this development photon pressure could be used to simply modify the orbit of a debris object enough to prevent a collision with an active satellite, rather than impart the significant change in orbit often required to return the debris to the atmosphere. Photon pressure can modify the orbit of a target by giving it momentum. While the total momentum imparted on the target is in the direction of the laser pulse, some of this will be along the orbit of the target, either slowing it down or speeding it up. Fig. 3 shows a schematic for a target at angle $Z$ from zenith, a distance $R$ away, with an altitude of $h_{t}$. Momentum $p_{p h}$ is imparted on the target through illumination, with a component $\vec{A}$ along the orbit. Using this schematic a simple relation between the laser power, target size and orbit displacement can be calculated. Fig. 4 shows a calculation of the relation between the number of passes required to move a target by $100 \mathrm{~m}$ and the diameter of the target, with a constant area to mass ratio of $0.0137 \mathrm{~m}^{2} \mathrm{~kg}^{-1}$.

If a collision is predicted the debris can then be moved enough to mitigate the risk of collision rather than expend limited and valuable propellant from the satellite itself. A laser in the class of tens of kilowatts would be required to achieve such a feat, but it would prove a worthy demonstration for the technology and its application. Deorbiting of space debris has been considered with project ORION ${ }^{14}$ demonstrating that a MW class laser could be used to modify a stable orbit into a decaying orbit with a $5 \mathrm{~m}$ telescope and AO correction. Such a system was considered very costly at the time due to the high power and large fast slewing telescope required.

AO technology for projecting a laser beam as so far only focused on improving the beam quality on the target. When tracking space debris at first it may not be obvious that correcting both the upward propagating beam and the off-axis reflection will be beneficial. Correcting the laser beam for propagation improves the Strehl at the target, increasing the flux on the target and thus increasing the number of photons reflected back toward the telescope. A single pixel detector can be used for ranging space debris as the system does not require an 


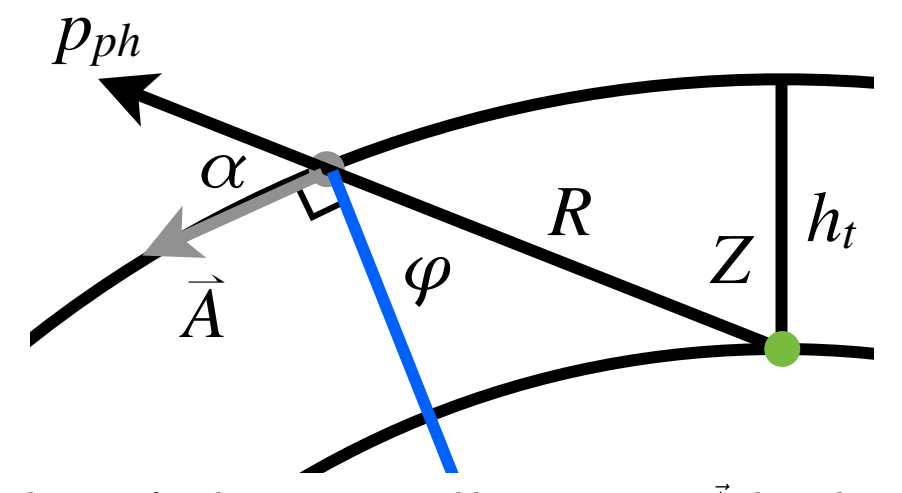

Figure 3. A schematic for photon pressure adding momentum $\vec{A}$ along the targets orbit.

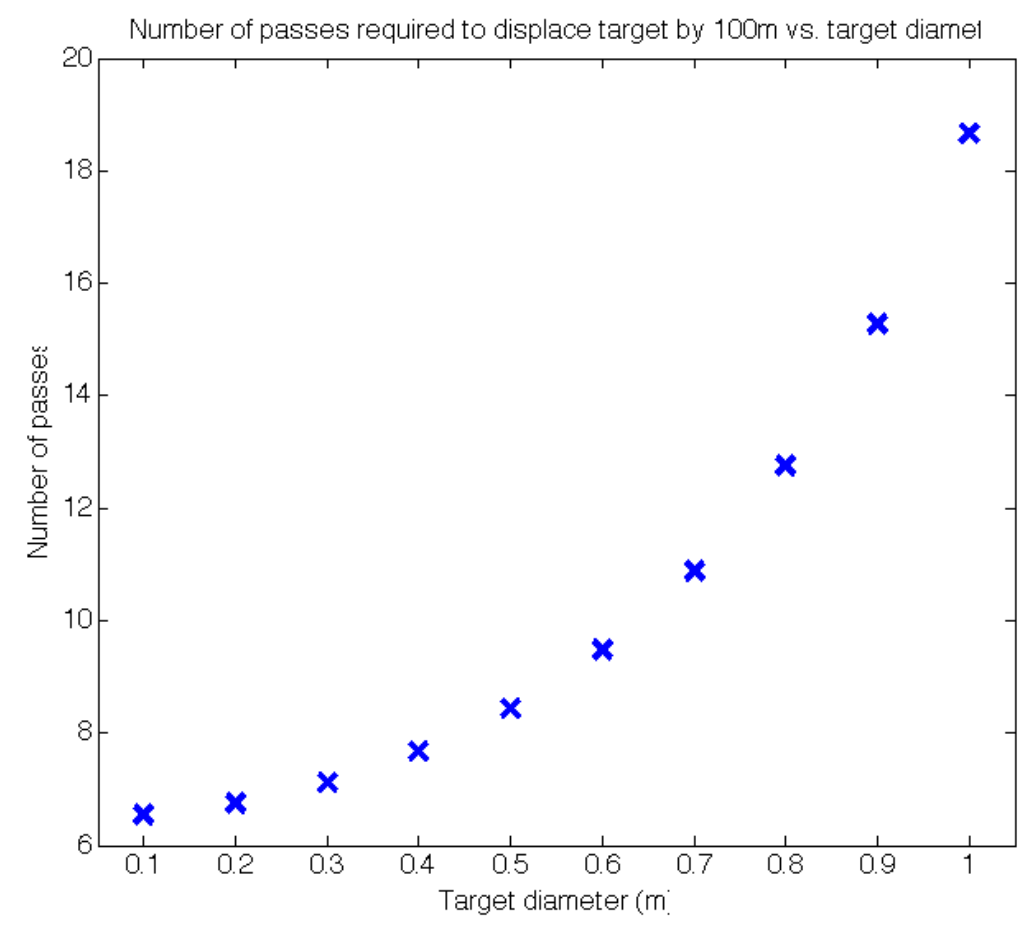

Figure 4. The number of passes of a target of varying diameter over a ground station to move the target by $100 \mathrm{~m}$ with a $10 \mathrm{~kW}$ ground station laser.

image of the debris to be captured. If the captured light is also AO corrected the intensity can be increased on the detector. The off-axis nature of the debris image further complicates matters, requiring a large field of correction or multiple deformable mirrors and guide stars.

\section{REFERENCES}

[1] Kessler, D. J. and Cour-Palais, B. G., "Collision Frequency of Artificial Satellites' The Creation of a Debris Belt," Journal of Geophysical Research 83, 2637 (1978).

[2] Liou, J.-C. and Johnson, N., "Characterization of the cataloged Fengyun-1C fragments and their long-term effect on the LEO environment," Advances in Space Research 43, 1407-1415 (May 2009).

[3] Liou, J.-C., "An active debris removal parametric study for LEO environment remediation," Advances in Space Research 47, 1865-1876 (June 2011). 
[4] Liou, J.-C. and Johnson, N., "Instability of the present LEO satellite populations," Advances in Space Research 41, 1046-1053 (Jan. 2008).

[5] Aslanov, V. and Yudintsev, V., "Dynamics of large space debris removal using tethered space tug," Acta Astronautica 91, 149-156 (2013).

[6] Gao, Y., Smith, C., and Greene, B., "Laser Tracking of Space Debris," in [European Space Surveillance Conference], (2011).

[7] Mason, J., Stupl, J., Marshall, W., and Levit, C., "Orbital debrisdebris collision avoidance," Advances in Space Research 48, 1643-1655 (Nov. 2011).

[8] Bennet, F., Conan, R., D’Ogreville, C., Dawson, M., Paulin, N., Price, I., Rigaut, F., Ritchie, I., Smith, C., and Uhlendorf, K., "Adaptive Optics For Laser Space Debris Removal," in [Adaptive Optics Systems III], 844744(1-6) (2012).

[9] Phipps, C. R., Baker, K. L., Libby, S. B., Liedahl, D. a., Olivier, S. S., Pleasance, L. D., Rubenchik, A., Trebes, J. E., Victor George, E., Marcovici, B., Reilly, J. P., and Valley, M. T., "Removing orbital debris with lasers," Advances in Space Research 49, 1283-1300 (May 2012).

[10] Liedahl, D. A., Rubenchik, A., Libby, S. B., Nikolaev, S., and Phipps, C. R., "Pulsed laser interactions with space debris : Target shape effects," Advances in Space Research 52(5), 895-915 (2013).

[11] Liou, J.-C. and Johnson, N. L., "A sensitivity study of the effectiveness of active debris removal in LEO," Acta Astronautica 64, 236-243 (Jan. 2009).

[12] Goldstein, R. M. G. S. J. and Kessler, D. J., "Radar observations of space debris," Planet. Space Sci. 46, 1007-1013 (1998).

[13] D’Orgeville, C., Bennet, F., Blundell, M., Brister, R., Chan, A., Dawson, M., Gao, Y., Paulin, N., Price, I., Rigaut, F., Ritchie, I., Sellars, M., Smith, C., Uhlendorf, K., and Wang, Y., "A Sodium Laser Guide Star Facility for the ANU/EOS Space Debris Tracking Adaptive Optics Demonstrator," in [These proc.], 9148-124 (2014).

[14] Campbell, J., "Project ORION: orbital debris removal using ground-based sensors and lasers," NASA Technical Memorandum (108522) (1997). 